Article

\title{
Ranking DMUs by Combining Cross-Efficiency Scores Based on Shannon's Entropy
}

\author{
Yueh-Chiang Lee \\ Department of Business Administration, Vanung University, Zhongli, Taoyuan 32061, Taiwan; \\ yclee@mail.vnu.edu.tw
}

Received: 1 April 2019; Accepted: 2 May 2019; Published: 4 May 2019

\begin{abstract}
Cross-efficiency evaluation is an effective approach for ranking decision-making units (DMUs), and there exist different perspectives from different cross-efficiency evaluation models. However, efficiency ranking results derived from cross-efficiency models may not be the same, and these models may provide some precious information that we cannot ignore. In this case, it may not be easy for one to decide which method should be used in some underlying assumptions, and we need several cross-efficiency evaluation models to measure simultaneously the cross-efficiency scores of DMUs. Hence, combining different viewpoints for ranking DMUs is a possible way to apply cross-efficiency evaluation. Since Shannon's entropy is an effective tool to measure uncertainty, in this study we adopt the idea of Shannon's entropy to combine cross-efficiency scores, which are obtained from different evaluation models, for comparison of DMUs. An example of commercial banks in Taiwan is used to illustrate the idea proposed in this paper.
\end{abstract}

Keywords: data envelopment analysis; cross efficiency; Shannon's entropy; ranking

\section{Introduction}

Data envelopment analysis (DEA) has been widely used as a powerful performance measurement tool to evaluate the efficiency of different comparable entities, namely decision-making units (DMUs) that consume multiple inputs to produce multiple outputs. Since each DMU measures its efficiency with the most favorable weights to itself, the nature of self-evaluation may lead to the case that many DMUs are evaluated as efficient and cannot be discriminated any further. In other words, DEA suffers from lack of discrimination power on efficient units, and the self-evaluation allows each DMU to be evaluated with its most favorable weights. The inputs and outputs will be heavily weighted for a favorable DMU, whereas those not favorable to the DMU will be less weighted. A commonly recognized problem of DEA is assessing too many units as efficient. In the literature DEA cross-efficiency evaluation has shown to be an effective methodology to improve the discrimination power among CCR efficient DMUs.

The DEA cross-efficiency method extends the self-evaluation into the peer-evaluation of conventional DEA models. The idea of cross-efficiency is to use the set of weights selected by each DMU in calculating its own efficiency as a specific set of common weights by which to calculate the efficiency of all other DMUs. The average of self- and peer-evaluation efficiency scores is treated as the overall cross-efficiency score of the DMU being evaluated. The cross-efficiency evaluation not only provides a ranking result among DMUs, but also removes unrealistic weight without the need of the elicitation of weight restrictions from experts [1]. Nevertheless, the conventional cross-efficiency evaluation still has some flaws. DEA models might exist with multiple optimum weights, which may result in different cross-efficiency scores. Under such a situation, we have different ranking results of DMUs. To tackle this problem, Sexton et al. [2] and Doyle and Green [3] introduced the secondary goals approach for the choice of weights among the alternative optimal solutions. Due to the sound property of the cross-efficiency evaluation, this approach has been applied to various industries, and a 
number of cross-efficiency models and applications have been reported in the literature (for examples, Liang et al. [4,5], Ramón et al. [6], Wang et al. [7], Wu et al. [8,9], Liu [10], Oukil [11], Al-Siyabi et al. [12], and Liu et al. [13]). Wu et al. [9] made a good review of the literature involving models, and Liu [10] described many novel applications.

Information entropy is an effective tool to measure the uncertainty. According to the idea of entropy, the amount or quality of information is one of the determinants for making decisions accurately. To this end, it has been widely applied to different cases of assessments, and there are also several articles that integrated the entropy and DEA models. Soleimani-Damaneh and Zarepisheh [14] employed Shannon's entropy to integrate a family of DEA efficiency scores, which are measured from different DEA models, into an efficiency index for ranking DMUs. Xie et al. [15] used Shannon's entropy to derive the degree of importance of each DMU. Then they merged the calculated efficiency scores and the degrees of importance to help discriminate traditional DEA models. Qi and Guo [16] proposed a modified weight-restricted DEA model for the derivation of non-zero optimal weights, where Shannon's entropy is used to aggregate those weights to be the common weights. Wang et al. [17] used the DEA entropy model to find the cross-efficiency intervals with imprecise inputs and outputs, and all DMUs are ranked according to the distance to ideal positive cross-efficiency. Lu and Liu [18] took into account the aggressive and benevolent formulations at the same time, and consequently, a number of cross-efficiency intervals are obtained for each DMU. The entropy is then used to construct a numerical index for ranking DMUs.

Different ways for determining the weights produce different cross efficiencies, and lead to different ranking results of DMUs [9,18]. In this case, it may be not easy for one to decide which method should be used in some underlying assumptions, and we need several cross-efficiency evaluation models to measure simultaneously the cross-efficiency scores of DMUs. In particular, each of the models and viewpoints might have some precious advantages that we cannot ignore. Zeleny [19] first proposed to apply Shannon's entropy as a coefficient of importance degree in multiple criteria decision analysis. As noted in Soleimani-Damaneh and Zarepisheh [14], if the outcomes of the performance analysis are important, one might try different models, from which to combine different results and viewpoints together. For this reason, in this study we adopt the idea of Soleimani-Damaneh and Zarepisheh [14] to calculate Shannon's entropy of the obtained cross-efficiencies from several cross-efficiency models for ranking DMUs.

In the sections that follow, we first introduce the cross-efficiency evaluation and the associated alternative secondary goal models. Then we develop the solution procedure to combine cross-efficiency scores from different evaluation models with Shannon's entropy for the comparison of DMUs. An example of commercial banks in Taiwan is applied to illustrate the ideal proposed in this study. Finally, some conclusions of this study are presented.

\section{Cross-Efficiency Evaluation}

Let $X_{i j}$ and $Y_{r j}$ denote the $i$-th input, $i=1, \ldots, m$, and $r$-th output, $r=1, \ldots, s$, respectively, of the $j$-th DMU, $j=1, \ldots, n$. The DEA model proposed by Charnes et al. [20] for calculating the efficiency of DMU $d$ under the assumption of constant returns-to-scale, referred to as the CCR model, is:

$$
\begin{gathered}
E_{d d}=\max \sum_{r=1}^{s} u_{r d} Y_{r d} \\
\text { s.t. } \sum_{i=1}^{m} v_{i d} X_{i d}=1 \\
\sum_{r=1}^{s} u_{r d} Y_{r j}-\sum_{i=1}^{m} v_{i d} X_{i j} \leq 0, j=1, \ldots, n, \\
u_{r d}, v_{i d} \geq 0, r=1, \ldots, s, i=1, \ldots, m,
\end{gathered}
$$


where $u_{r d}$ and $v_{i d}$ are the weights assigned to the $s$ outputs and $m$ inputs, respectively.

In the cross-efficiency evaluation we use the optimal weights obtained from (1) to calculate the cross-efficiency scores. Specifically, if $v_{i d}^{*}(i=1, \ldots, m)$ and $u_{r d}^{*}(r=1, \ldots, s)$ is an optimal solution of (1) for a given DMU $d$, then the cross-efficiency of $\operatorname{DMU} j(j=1, \ldots, n, j \neq d)$ peer-evaluated by DMU $d$ is given by:

$$
E_{d j}=\frac{\sum_{r=1}^{s} u_{r d}^{*} Y_{r j}}{\sum_{i=1}^{m} v_{i d}^{*} X_{i j}}, d, j=1, \ldots, n
$$

The cross-efficiency score of $\mathrm{DMU} j, j=1, \ldots, n$, is calculated as the average of its cross-efficiencies obtained with the weights of all the DMUs. In other words, the cross-efficiency of DMU $j$ is defined as:

$$
\bar{E}_{j}=\frac{1}{n} \sum_{d=1}^{n} E_{d j}, j=1, \ldots, n
$$

\section{Alternative Secondary Goal Models}

The cross-efficiency scores calculated from DEA models may not be unique because of the multiple optimal solutions for DEA weights, and we obtain different efficiencies with different solutions of DEA weights. To eliminate the non-uniqueness, Sexton et al. [2] and Doyle and Green [3] proposed to use secondary goals to choose the weights among the optimal solutions. Since then a number of cross-efficiency models and applications have been reported in the literature. From the record of Web-of-Science, the first three most cited articles in cross-efficiency study are, namely Doyle and Green [3] and Liang et al. [4,5]. Therefore, in this paper we employ these three studies to measure cross-efficiency scores of DMUs, respectively, and combine their obtained results together to calculate Shannon's entropy for comparison of DMUs.

\subsection{The Method of Secondary Goals}

The most commonly used secondary goals approach is proposed by Doyle and Green [3]. They defined the aggregate efficiency to be the weighted average of the other $n-1$ efficiencies, with the weight of $\sum_{i=1}^{m} v_{i d} X_{i d} / \sum_{j=1, j \neq d}^{n} \sum_{i=1}^{m} v_{i d} X_{i j}$ for DMU $d$ to obtain the following model:

$$
\begin{gathered}
\max \frac{\sum_{j=1, j \neq d}^{n} \sum_{r=1}^{s} u_{r d} Y_{r d}}{\sum_{j=1, j \neq d}^{n} \sum_{i=1}^{m} v_{i d} X_{i d}} \\
\text { s.t. } \sum_{r=1}^{s} u_{r d} Y_{r d}=E_{d d} \sum_{i=1}^{m} v_{i d} X_{i d} \\
\sum_{r=1}^{s} u_{r d} Y_{r j}-\sum_{i=1}^{m} v_{i d} X_{i j} \leq 0, j=1, \ldots, n \\
u_{r d}, v_{i d} \geq 0, r=1, \ldots, s, I=1, \ldots, m
\end{gathered}
$$

where $E_{d d}$ is the CCR efficiency of DMU $d$ obtained from (1). 
This model is a linear fractional program, which can be linearized by applying the variable substitution technique of Charnes and Cooper [21] as follows:

$$
\begin{gathered}
\max \sum_{j=1, j \neq d}^{n} \sum_{r=1}^{s} u_{r d} Y_{r j} \\
\text { s.t. } \sum_{j=1, j \neq d}^{n} \sum_{i=1}^{m} v_{i d} X_{i d}=1 \\
\sum_{r=1}^{s} u_{r d} Y_{r d}=E_{d d} \sum_{i=1}^{m} v_{i d} X_{i d} \\
\sum_{r=1}^{s} u_{r d} Y_{r j}-\sum_{i=1}^{m} v_{i d} X_{i j} \leq 0, j=1, \ldots, n \\
u_{r d}, v_{i d} \geq 0, r=1, \ldots, s, i=1, \ldots, m
\end{gathered}
$$

Model (5) is known as the benevolent formulation for cross-efficiency evaluation that aims to maximize the cross-efficiency scores of the other $n-1$ DMUs to some extent. The set of weights obtained from this model may not be the same as that obtained from Model (1). However, due to the constraint of " $\sum_{r=1}^{s} u_{r d} Y_{r d}=E_{d d} \sum_{i=1}^{m} v_{i d} X_{i d}$ ", it will produce the same efficiency $E_{d d}$ for DMU $d$. When Model (5) performs $n$ time, we have $n$ different sets of optimal soltions $v_{i d}^{*}$ and $u_{r d^{\prime}}^{*}$ and these optimal weights sets are used to calculate the cross-efficiency for each DMU by Equations (2) and (3).

\subsection{The Method of Mean Absolute Deviation}

Liang et al. [4] pointed out that Model (1) can be represented equivalently in the following deviation variable form:

$$
\begin{gathered}
\min s_{d} \\
\text { s.t. } \sum_{r=1}^{s} v_{i d} X_{i d}=1 \\
\sum_{r=1}^{s} u_{r d} Y_{r j}-\sum_{r=1}^{s} v_{i d} X_{i j}+s_{j}=0, j=1, \ldots, n \\
u_{r d}, v_{i d}, s_{j} \geq 0, r=1, \ldots, s, i=1, \ldots, m, j=1, \ldots, n .
\end{gathered}
$$

where $s_{d}$ and $s_{j}$ are the deviation variables for DMUs $d$ and $j$, respectively. Under this model, if $s_{d}^{*}=0$, then DMU $d$ is efficient. Otherwise, the efficiency score of DMU $d$ is $E_{d d}=1-s_{d}^{*}$. Liang et al. [4] referred to this deviation variable $s_{j}$ as the $d$-inefficiency of DMU $j$.

To search for the minimization of the variation among DMUs, they proposed the following secondary goal model:

$$
\begin{gathered}
\min \frac{1}{n} \sum_{j=1}^{n}\left|s_{j}-\bar{s}_{j}\right| \\
\text { s.t. } \sum_{r=1}^{s} v_{i d} X_{i d}=1 \\
\sum_{r=1}^{s} u_{r d} Y_{r d}=1-s_{d}^{*} \\
\sum_{r=1}^{s} u_{r d} Y_{r j}-\sum_{r=1}^{s} v_{i d} X_{i j}+s_{j}=0, j=1, \ldots, n \\
u_{r d}, v_{i d}, s_{j} \geq 0, r=1, \ldots, s, i=1, \ldots, m, j=1, \ldots, n .
\end{gathered}
$$


where $\bar{s}_{j}=1 / n \sum_{j=1}^{n} s_{j}$.

Let $\alpha_{j}=\frac{1}{2}\left(\left|s_{j}-\bar{s}_{j}\right|+\left(s_{j}-\bar{s}_{j}\right)\right)$ and $\beta_{j}=\frac{1}{2}\left(\left|s_{j}-\bar{s}_{j}\right|-\left(s_{j}-\bar{s}_{j}\right)\right)$. Model (7) can be transformed into the following linear program:

$$
\begin{gathered}
\min \frac{1}{n} \sum_{j=1}^{n}\left(\alpha_{j}+\beta_{j}\right) \\
\text { s.t. } \sum_{r=1}^{s} v_{i d} X_{i d}=1 \\
\sum_{r=1}^{s} u_{r d} Y_{r d}=E_{d d} \\
\sum_{r=1}^{s} u_{r d} Y_{r j}-\sum_{r=1}^{s} v_{i d} X_{i j}+s_{j}=0, j=1, \ldots, n \\
\alpha_{j}-\beta_{j}=s_{j}-\frac{1}{n} \sum_{j=1}^{n} s_{j}, j=1, \ldots, n \\
u_{r d}, v_{i d}, s_{j}, \alpha_{j}, \beta_{j} \geq 0, r=1, \ldots, s, i=1, \ldots, m, j=1, \ldots, n .
\end{gathered}
$$

Similar to (1), the cross-efficiency $\bar{E}_{j}$ for DMU $j$ is calculated through Equations (2) and (3).

\subsection{The Method of Game Cross-Efficiency}

Liang et al. [5] viewed each DMU as a player that searched for the maximization of its own efficiency, under the condition that the cross efficiency of each of the other DMUs does not deteriorate. Based on the idea of Liang et al. [5], the cross-efficiency of DMU $d$ relative to DMU $j$ can be attained through the following linear program:

$$
\begin{gathered}
E_{d j}=\max \sum_{r=1}^{s} u_{r d} Y_{r j} \\
\text { s.t. } \sum_{i=1}^{m} v_{i d} X_{i j}=1, \\
\sum_{r=1}^{s} u_{r d} Y_{r d}-E_{d d} \sum_{i=1}^{m} v_{i d} X_{i d}=0, \\
\sum_{r=1}^{s} u_{r d} Y_{r d}-\sum_{i=1}^{m} v_{i d} X_{i j} \leq 0, j=1, \ldots, n, j \neq d, \\
u_{r d}, v_{i d} \geq 0, r=1, \ldots, s, i=1, \ldots, m .
\end{gathered}
$$

In Equation (9) DMU $j$ seeks to maximize its own cross-efficiency score under the condition of maintaining the self-evaluation efficiency of DMU $d$ unchanged. The optimal solution $E_{d j}^{*}$ is the cross-efficiency of DMU $j$ peer-evaluated by DMU $d$, and the cross-efficiency of DMU $j$ is calculated as $\bar{E}_{j}=\frac{1}{n} \sum_{d=1}^{n} E_{d j}, j=1, \ldots, n$, which is defined in Equation (3).

\section{Combination of Cross-Efficiencies with Shannon's Entropy}

Shannon's entropy plays a crucial role that has an important impact on information theory [22]. Due to the sound property of the entropy, it has been now widely employed to assess importance or uncertainty in theoretical and application studies. With the basis of this idea, Soleimani-Damaneh and Zarepisheh [14] proposed a solution procedure to obtain the importance degree from different 
DEA models, and an efficiency index is calculated by the combination of the derived efficiencies for ranking DMUs. In this study the idea of Soleimani-Damaneh and Zarepisheh [14] is adopted as a tool to calculate Shannon's entropy for ranking DMUs with the cross-efficiency scores obtained from different evaluation models.

Let $n$ and $q$ denote the numbers of DMUs and cross-efficiency evaluation models, respectively, and the obtained cross-efficiency results are represented as the matrix $E_{n \times q}$. Each row of $E$ stands for a DMU and each of its columns corresponds to a cross-efficiency evaluation model. In other words, $E_{j \times p}$ represents the cross- efficiency of DMU $j, j=1, \ldots, n$, derived by a cross-efficiency evaluation model $C_{p}$ for $p=1, \ldots, q$. With the cross-efficiency scores evaluated from different models, the approach of Shannon's entropy for ranking DMUs can be summarized as the following algorithm.

Step 0. Calculate the CCR efficiency scores for all DMUs by using Model (1).

Step 1. Calculate the cross-efficiency matrix $E_{n \times q}$.

$$
E=\left[\begin{array}{cccc}
E_{11} & E_{12} & \cdots & E_{1 q} \\
E_{21} & E_{22} & \cdots & E_{2 q} \\
E_{31} & E_{32} & \cdots & E_{3 q} \\
\vdots & \vdots & & \vdots \\
E_{n 1} & E_{n 2} & \cdots & E_{n q}
\end{array}\right]
$$

Step 2. Normalize the cross-efficiency matrix by setting

$$
\hat{E}_{j p}=\frac{E_{j p}}{\sum_{j=1}^{n} E_{j p}}, j=1, \ldots, n, p=1, \ldots, q .
$$

Step 3. Compute Shannon's entropy $H_{p}$ for each cross-efficiency evaluation model as $H_{p}=$ $-(\ln n)^{-1} \sum_{j=1}^{n} \hat{E}_{j p} \ln \hat{E}_{j p}, p=1, \ldots, q$, where $(\ln n)^{-1}$ is the entropy constant.

Step 4 . Set $D_{p}=1-H_{p}$ as the degree of diversification for each cross-efficiency evaluation model.

Step 5. Calculate the degree of importance for model $C_{p}$, and let $w_{p}=\frac{D_{p}}{\sum_{p=1}^{q} D_{p}}, p=1, \ldots, q$, as the weight coefficient of model $C_{p}$.

Step 6. Calculate the composite cross-efficiency scores $E_{j}^{C *}=\sum_{p=1}^{q} w_{p} E_{j p}, j=1, \ldots, n$, for comparison of DMUs. The larger the value of $E_{j}^{C *}$ the better the DMU is.

\section{Example}

Liu [10] investigated the cross-efficiency scores of 22 commercial banks in Taiwan. Three inputs and three outputs are considered in measuring cross-efficiencies: the labor cost $\left(X_{1}\right)$, the physical capital $\left(X_{2}\right)$, and the purchase funds $\left(X_{3}\right)$ as the inputs, and the demand deposits $\left(Y_{1}\right)$, the short-term loans $\left(Y_{2}\right)$, and the medium- and long-term loans $\left(Y_{3}\right)$ as the outputs. Data for the 22 banks are shown in Table 1. We use this dataset to illustrate how our model is applied to calculate the composite cross-efficiency scores with Shannon's entropy for ranking the commercial banks. We first measure CCR efficiency scores for the 22 commercial banks, and their results are listed in the last column of Table 1. 
Table 1. Real data (in millions of Taiwan dollars) and CCR efficiency scores of 22 commercial banks in Taiwan.

\begin{tabular}{cccccccc}
\hline Bank & Labor & Capital & $\begin{array}{c}\text { Purchased } \\
\text { Funds }\end{array}$ & Deposits & $\begin{array}{c}\text { S-Term } \\
\text { Loans }\end{array}$ & $\begin{array}{c}\text { ML-Term } \\
\text { Loans }\end{array}$ & CCR \\
\hline 1 & 9492 & 23,935 & $1,029,108$ & 336,735 & 297,352 & 844,783 & 0.9327 \\
2 & 848 & 2683 & 121,212 & 24,362 & 27,961 & 79,582 & 0.8496 \\
3 & 2351 & 3416 & 323,449 & 106,247 & 104,348 & 259,497 & 1.0000 \\
4 & 7306 & 14,299 & 815,246 & 279,769 & 339,261 & 617,217 & 1.0000 \\
5 & 1388 & 2744 & 162,563 & 23,395 & 69,956 & 108,206 & 1.0000 \\
6 & 1999 & 6195 & 125,917 & 15,016 & 30,227 & 69,487 & 0.6306 \\
7 & 2838 & 7644 & 307,145 & 56,564 & 71,591 & 158,042 & 0.6205 \\
8 & 3545 & 2814 & 325,073 & 48,824 & 48,539 & 247,323 & 0.9050 \\
9 & 3585 & 3343 & 280,959 & 56,041 & 64,251 & 202,585 & 0.8058 \\
10 & 1775 & 1128 & 204,472 & 21,517 & 36,705 & 150,177 & 0.9419 \\
11 & 10,717 & 28,674 & $1,226,897$ & 508,605 & 384,511 & $1,023,549$ & 1.0000 \\
12 & 9308 & 11,294 & $1,078,604$ & 250,407 & 310,403 & 783,664 & 0.8654 \\
13 & 9346 & 22,617 & $1,271,363$ & 336,838 & 289,442 & 744,008 & 0.7767 \\
14 & 6455 & 18,487 & 841,496 & 305,603 & 187,843 & 643,889 & 1.0000 \\
15 & 3074 & 2150 & 395,750 & 66,537 & 92,533 & 307,930 & 1.0000 \\
16 & 12,502 & 14,519 & $1,347,592$ & 580,389 & 462,928 & $1,188,269$ & 1.0000 \\
17 & 9277 & 17,464 & 715,304 & 163,804 & 158,695 & 547,688 & 0.7958 \\
18 & 3642 & 6915 & 505,286 & 105,395 & 103,643 & 341,020 & 0.8481 \\
19 & 8049 & 11,002 & 616,242 & 232,732 & 218,083 & 594,174 & 1.0000 \\
20 & 20,295 & 34,229 & 997,936 & 146,904 & 348,395 & 941,957 & 0.9831 \\
21 & 11,405 & 27,730 & $1,243,848$ & 476,748 & 404,671 & $1,028,704$ & 0.9453 \\
22 & 14,354 & 38,694 & $1,825,537$ & 442,195 & 376,648 & $1,531,299$ & 1.0000 \\
\hline
\end{tabular}

Following Step 1 of the algorithm introduced in Section 4, we employ Models (5), (8) and (9) to calculate the cross-efficiency scores of the commercial banks, respectively, with the results shown in Tables 2-4. The elements of the cross-efficiency matrix $E_{n \times q}$, which stem from the last rows of Tables 2-4, are listed in Table 5. The number in the parenthesis of Table 5 is the ranking place of the commercial bank. It can be found in Table 5 that inconsistent ranking occurs for banks under different models. For example, Bank no. 4 has fifth place under the evaluation of Models (5) and (8), but is ranked as third place under Model (9). From the practical standpoint, the combination of the results obtained from the three models seems to be a reasonable method for comparison of the commercial banks. 
Table 2. The cross-efficiency of 22 Taiwanese commercial banks via Doyle and Green [3].

\begin{tabular}{|c|c|c|c|c|c|c|c|c|c|c|c|c|c|c|c|c|c|c|c|c|c|c|}
\hline Bank & 1 & 2 & 3 & 4 & 5 & 6 & 7 & 8 & 9 & 10 & 11 & 12 & 13 & 14 & 15 & 16 & 17 & 18 & 19 & 20 & 21 & 22 \\
\hline 1 & 0.9327 & 0.8076 & 0.9771 & 0.8680 & 0.7745 & 0.5105 & 0.5843 & 0.8173 & 0.7306 & 0.8502 & 0.9640 & 0.8427 & 0.7105 & 0.9183 & 0.9306 & 1.0000 & 0.7709 & 0.8238 & 0.9680 & 0.7764 & 0.9414 & 0.9997 \\
\hline 2 & 0.8062 & 0.8496 & 1.0000 & 0.7653 & 0.7061 & 0.3149 & 0.5044 & 0.6320 & 0.5119 & 0.7662 & 0.8651 & 0.7627 & 0.7211 & 0.9036 & 0.9075 & 0.8610 & 0.5348 & 0.8481 & 0.6687 & 0.4204 & 0.8170 & 0.9663 \\
\hline 3 & 0.9296 & 0.8248 & 1.0000 & 0.8850 & 0.8008 & 0.4866 & 0.5883 & 0.7890 & 0.7033 & 0.8428 & 0.9680 & 0.8489 & 0.7255 & 0.9249 & 0.9371 & 1.0000 & 0.7388 & 0.8352 & 0.9344 & 0.7172 & 0.9428 & 1.0000 \\
\hline 4 & 0.8973 & 0.7476 & 0.9562 & 1.0000 & 0.9592 & 0.5835 & 0.6205 & 0.6781 & 0.7139 & 0.7215 & 0.9425 & 0.8392 & 0.6875 & 0.8077 & 0.8277 & 1.0000 & 0.7333 & 0.7282 & 1.0000 & 0.8732 & 0.9433 & 0.8381 \\
\hline 5 & 0.8356 & 0.7796 & 1.0000 & 1.0000 & 1.0000 & 0.4385 & 0.5969 & 0.5470 & 0.5686 & 0.6585 & 0.9058 & 0.8164 & 0.7126 & 0.7929 & 0.8115 & 0.9414 & 0.5732 & 0.7368 & 0.8032 & 0.5735 & 0.8936 & 0.7977 \\
\hline 6 & 0.8322 & 0.6649 & 0.8758 & 1.0000 & 0.9793 & 0.6306 & 0.6024 & 0.5872 & 0.6920 & 0.6218 & 0.8764 & 0.7863 & 0.6269 & 0.7041 & 0.7266 & 0.9454 & 0.7022 & 0.6338 & 1.0000 & 0.9831 & 0.8916 & 0.7122 \\
\hline 7 & 0.8973 & 0.7476 & 0.9562 & 1.0000 & 0.9592 & 0.5835 & 0.6205 & 0.6781 & 0.7139 & 0.7215 & 0.9425 & 0.8392 & 0.6875 & 0.8077 & 0.8277 & 1.0000 & 0.7333 & 0.7282 & 1.0000 & 0.8732 & 0.9433 & 0.8381 \\
\hline 8 & 0.7303 & 0.5957 & 0.9141 & 0.7474 & 0.6655 & 0.3391 & 0.4453 & 0.9050 & 0.7980 & 0.9419 & 0.7408 & 0.8295 & 0.5749 & 0.6963 & 1.0000 & 1.0000 & 0.6679 & 0.7193 & 0.9461 & 0.7053 & 0.7483 & 0.7738 \\
\hline 9 & 0.7992 & 0.6475 & 0.9124 & 0.7882 & 0.6986 & 0.4152 & 0.4919 & 0.8876 & 0.8058 & 0.8950 & 0.8112 & 0.8273 & 0.6074 & 0.7560 & 0.9494 & 1.0000 & 0.7358 & 0.7371 & 1.0000 & 0.8168 & 0.8141 & 0.8362 \\
\hline 10 & 0.7303 & 0.5957 & 0.9141 & 0.7473 & 0.6655 & 0.3391 & 0.4452 & 0.9050 & 0.7980 & 0.9419 & 0.7408 & 0.8295 & 0.5749 & 0.6962 & 1.0000 & 1.0000 & 0.6678 & 0.7193 & 0.9461 & 0.7053 & 0.7482 & 0.7738 \\
\hline 11 & 0.8445 & 0.7384 & 1.0000 & 0.8484 & 0.5681 & 0.2850 & 0.5029 & 0.5211 & 0.4888 & 0.5500 & 1.0000 & 0.7123 & 0.7603 & 0.9871 & 0.7129 & 1.0000 & 0.5282 & 0.7460 & 0.7443 & 0.3570 & 0.9209 & 0.8462 \\
\hline 12 & 0.5076 & 0.4353 & 1.0000 & 0.8981 & 0.9609 & 0.2191 & 0.3892 & 0.4681 & 0.5812 & 0.7379 & 0.5527 & 0.8654 & 0.5012 & 0.4191 & 0.9995 & 1.0000 & 0.3567 & 0.5417 & 0.7023 & 0.4043 & 0.5902 & 0.3966 \\
\hline 13 & 0.7861 & 0.6859 & 1.0000 & 0.8379 & 0.4766 & 0.2056 & 0.4600 & 0.3754 & 0.3841 & 0.3837 & 1.0000 & 0.6378 & 0.7767 & 1.0000 & 0.5796 & 0.9832 & 0.4215 & 0.6850 & 0.6447 & 0.2257 & 0.8963 & 0.7391 \\
\hline 14 & 0.8116 & 0.7115 & 1.0000 & 0.8342 & 0.5043 & 0.2351 & 0.4755 & 0.4418 & 0.4273 & 0.4605 & 1.0000 & 0.6687 & 0.7701 & 1.0000 & 0.6419 & 0.9891 & 0.4664 & 0.7164 & 0.6836 & 0.2757 & 0.9055 & 0.7934 \\
\hline 15 & 0.8329 & 0.7377 & 1.0000 & 0.8168 & 0.7355 & 0.3773 & 0.5145 & 0.8210 & 0.7028 & 0.9020 & 0.8622 & 0.8506 & 0.6751 & 0.8366 & 1.0000 & 1.0000 & 0.6716 & 0.8166 & 0.8862 & & 0.8480 & 0.9163 \\
\hline 16 & 0.9322 & 0.7890 & 0.9574 & 0.8654 & 0.7689 & 0.5381 & 0.5841 & 0.8295 & 0.7530 & 0.8453 & 0.9590 & 0.8374 & 0.6968 & 0.9036 & 0.9166 & 1.0000 & 0.7958 & 0.8066 & 1.0000 & 0.8408 & 0.9404 & 0.9857 \\
\hline 17 & 0.9322 & 0.7890 & 0.9574 & 0.8654 & 0.7689 & 0.5381 & 0.5841 & 0.8295 & 0.7529 & 0.8454 & 0.9590 & 0.8374 & 0.6968 & 0.9036 & 0.9166 & 1.0000 & 0.7958 & 0.8066 & 1.0000 & 0.8407 & 0.9404 & 0.9857 \\
\hline 18 & 0.8064 & 0.8496 & 1.0000 & 0.7654 & 0.7062 & 0.3151 & 0.5045 & 0.6322 & 0.5121 & 0.7663 & 0.8653 & 0.7628 & 0.7211 & 0.9036 & 0.9075 & 0.8611 & 0.5350 & 0.8481 & 0.6690 & 0.4207 & 0.8172 & 0.9664 \\
\hline 19 & 0.9322 & 0.7890 & 0.9574 & 0.8654 & 0.7689 & 0.5381 & 0.5841 & 0.8295 & 0.7530 & 0.8453 & 0.9590 & 0.8374 & 0.6968 & 0.9036 & 0.9166 & 1.0000 & 0.7958 & 0.8066 & 1.0000 & 0.8408 & 0.9404 & 0.9857 \\
\hline 20 & 0.8323 & 0.6650 & 0.8756 & 0.9988 & 0.9778 & 0.6303 & 0.6020 & 0.5883 & 0.6923 & 0.6226 & 0.8764 & 0.7862 & 0.6268 & 0.7046 & 0.7270 & 0.9452 & 0.7027 & 0.6342 & 1.0000 & 0.9831 & 0.8914 & 0.7131 \\
\hline 21 & 0.9001 & 0.7889 & 1.0000 & 0.9999 & 0.9648 & 0.5175 & 0.6191 & 0.6638 & 0.6700 & 0.7369 & 0.9542 & 0.8508 & 0.7180 & 0.8416 & 0.8601 & 1.0000 & 0.6868 & 0.7667 & 0.9311 & 0.7310 & 0.9453 & 0.8720 \\
\hline 22 & 0.9327 & 0.8080 & 0.9775 & 0.8680 & 0.7746 & 0.5100 & 0.5843 & 0.8170 & 0.7301 & 0.8503 & 0.9641 & 0.8428 & 0.7108 & 0.9186 & 0.9308 & 1.0000 & & 0.8241 & 0.9674 & 0.7752 & 0.9414 & 1.0000 \\
\hline Ave. & 0.8382 & 0.7294 & 0.9651 & 0.8757 & 0.7811 & 0.4341 & 0.5411 & 0.6929 & 0.6583 & 0.7503 & 0.8959 & 0.8050 & 0.6809 & 0.8332 & 0.8649 & 0.9785 & 0.6538 & 0.7504 & 0.8861 & 0.6711 & 0.8755 & 0.8516 \\
\hline
\end{tabular}


Table 3. The cross-efficiency of 22 Taiwanese commercial banks via Liang et al. [4].

\begin{tabular}{|c|c|c|c|c|c|c|c|c|c|c|c|c|c|c|c|c|c|c|c|c|c|c|}
\hline Bank & 1 & 2 & 3 & 4 & 5 & 6 & 7 & 8 & 9 & 10 & 11 & 12 & 13 & 14 & 15 & 16 & 17 & 18 & 19 & 20 & 21 & 22 \\
\hline 1 & 0.9327 & 0.8076 & 0.9771 & 0.8680 & 0.7745 & 0.5105 & 0.5843 & 0.8173 & 0.7306 & 0.8502 & 0.9640 & 0.8427 & 0.7105 & 0.9183 & 0.9306 & 1.0000 & 0.7709 & 0.8238 & 0.9680 & 0.7764 & 0.9414 & 0.9997 \\
\hline 2 & 0.8062 & 0.8496 & 1.0000 & 0.7653 & 0.7061 & 0.3149 & 0.5044 & 0.6320 & 0.5119 & 0.7662 & 0.8651 & 0.7627 & 0.7211 & 0.9036 & 0.9075 & 0.8610 & 0.5348 & 0.8481 & 0.6687 & 0.4204 & 0.8170 & 0.9663 \\
\hline 3 & 0.9161 & 0.8083 & 1.0000 & 0.9375 & 0.8758 & 0.5005 & 0.6024 & 0.7321 & 0.6882 & 0.7944 & 0.9617 & 0.8497 & 0.7220 & 0.8867 & 0.9018 & 1.0000 & 0.7153 & 0.8037 & 0.9329 & 0.7234 & 0.9439 & 0.9413 \\
\hline 4 & 0.8973 & 0.7476 & 0.9562 & 1.0000 & 0.9592 & 0.5835 & 0.6205 & 0.6781 & 0.7139 & 0.7215 & 0.9425 & 0.8392 & 0.6875 & 0.8077 & 0.8277 & 1.0000 & 0.7333 & 0.7282 & 1.0000 & 0.8732 & 0.9433 & 0.8381 \\
\hline 5 & 0.8023 & 0.6800 & 0.8975 & 1.0000 & 1.0000 & 0.5265 & 0.5906 & 0.5205 & 0.6069 & 0.5924 & 0.8597 & 0.7763 & 0.6396 & 0.6978 & 0.7198 & 0.9167 & 0.6089 & 0.6379 & 0.8798 & 0.7391 & 0.8675 & 0.6937 \\
\hline 6 & 0.8322 & 0.6649 & 0.8758 & 1.0000 & 0.9793 & 0.6306 & 0.6024 & 0.5872 & 0.6920 & 0.6218 & 0.8764 & 0.7863 & 0.6269 & 0.7041 & 0.7266 & 0.9454 & 0.7022 & 0.6338 & 1.0000 & 0.9831 & 0.8916 & 0.7122 \\
\hline 7 & 0.8973 & 0.7476 & 0.9562 & 1.0000 & 0.9592 & 0.5835 & 0.6205 & 0.6781 & 0.7139 & 0.7215 & 0.9425 & 0.8392 & 0.6875 & 0.8077 & 0.8277 & 1.0000 & 0.7333 & 0.7282 & 1.0000 & 0.8732 & 0.9433 & 0.8381 \\
\hline 8 & 0.7303 & 0.5957 & 0.9141 & 0.7474 & 0.6655 & 0.3391 & 0.4453 & 0.9050 & 0.7980 & 0.9419 & 0.7408 & 0.8295 & 0.5749 & 0.6963 & 1.0000 & 1.0000 & 0.6679 & 0.7193 & 0.9461 & 0.7053 & 0.7483 & 0.7738 \\
\hline 9 & 0.7992 & 0.6475 & 0.9124 & 0.7882 & 0.6986 & 0.4152 & 0.4919 & 0.8876 & 0.8058 & 0.8950 & 0.8112 & 0.8273 & 0.6074 & 0.7560 & 0.9494 & 1.0000 & 0.7358 & 0.7371 & 1.0000 & 0.8168 & 0.8141 & 0.8362 \\
\hline 10 & 0.7303 & 0.5957 & 0.9141 & 0.7473 & 0.6655 & 0.3391 & 0.4452 & 0.9050 & 0.7980 & 0.9419 & 0.7408 & 0.8295 & 0.5749 & 0.6962 & 1.0000 & 1.0000 & 0.6678 & 0.7193 & 0.9461 & 0.7053 & 0.7482 & 0.7738 \\
\hline 11 & 0.8445 & 0.7384 & 1.0000 & 0.8484 & 0.5681 & 0.2850 & 0.5029 & 0.5211 & 0.4888 & 0.5500 & 1.0000 & 0.7123 & 0.7603 & 0.9871 & 0.7129 & 1.0000 & 0.5282 & 0.7460 & 0.7443 & 0.3570 & 0.9209 & 0.8462 \\
\hline 12 & 0.5076 & 0.4353 & 1.0000 & 0.8981 & 0.9609 & 0.2191 & 0.3892 & 0.4681 & 0.5812 & 0.7379 & 0.5527 & 0.8654 & 0.5012 & 0.4191 & 0.9995 & 1.0000 & 0.3567 & 0.5417 & 0.7023 & 0.4043 & 0.5902 & 0.3966 \\
\hline 13 & 0.7861 & 0.6859 & 1.0000 & 0.8379 & 0.4766 & 0.2056 & 0.4600 & 0.3754 & 0.3841 & 0.3837 & 1.0000 & 0.6378 & 0.7767 & 1.0000 & 0.5796 & 0.9832 & 0.4215 & 0.6850 & 0.6447 & 0.2257 & 0.8963 & 0.7391 \\
\hline 14 & 0.8116 & 0.7115 & 1.0000 & 0.8342 & 0.5043 & 0.2351 & 0.4755 & 0.4418 & 0.4273 & 0.4605 & 1.0000 & 0.6687 & 0.7701 & 1.0000 & 0.6419 & 0.9891 & 0.4664 & 0.7164 & 0.6836 & 0.2757 & 0.9055 & 0.7934 \\
\hline 15 & 0.8329 & 0.7377 & 1.0000 & 0.8168 & 0.7355 & 0.3773 & 0.5145 & 0.8210 & 0.7028 & 0.9020 & 0.8622 & 0.8506 & 0.6751 & 0.8366 & 1.0000 & 1.0000 & 0.6716 & 0.8166 & 0.8862 & & 0.8480 & 0.9163 \\
\hline 16 & 0.9237 & 0.7787 & 0.9571 & 0.8983 & 0.8155 & 0.5489 & 0.5930 & 0.7928 & 0.7436 & 0.8150 & 0.9549 & 0.8378 & 0.6945 & 0.8800 & 0.8947 & 1.0000 & 0.7808 & 0.7873 & 1.0000 & 0.8483 & 0.9411 & 0.9494 \\
\hline 17 & 0.9317 & 0.7882 & 0.9566 & 0.8648 & 0.7684 & 0.5383 & 0.5838 & 0.8293 & 0.7529 & 0.8448 & 0.9584 & 0.8368 & 0.6962 & 0.9029 & 0.9159 & 0.9995 & 0.7958 & 0.8059 & 1.0000 & 0.8414 & 0.9399 & 0.9850 \\
\hline 18 & 0.8064 & 0.8496 & 1.0000 & 0.7654 & 0.7062 & 0.3151 & 0.5045 & 0.6322 & 0.5121 & 0.7663 & 0.8653 & 0.7628 & 0.7211 & 0.9036 & 0.9075 & 0.8611 & 0.5350 & 0.8481 & 0.6690 & 0.4207 & 0.8172 & 0.9664 \\
\hline 19 & 0.9199 & 0.7732 & 0.9485 & 0.8870 & 0.8017 & 0.5490 & 0.5880 & 0.7969 & 0.7449 & 0.8150 & 0.9494 & 0.8321 & 0.6888 & 0.8769 & 0.8913 & 0.9944 & 0.7834 & 0.7835 & 1.0000 & 0.8544 & 0.9356 & 0.9483 \\
\hline 20 & 0.8323 & 0.6650 & 0.8756 & 0.9988 & 0.9778 & 0.6303 & 0.6020 & 0.5883 & 0.6923 & 0.6226 & 0.8764 & 0.7862 & 0.6268 & 0.7046 & 0.7270 & 0.9452 & 0.7027 & 0.6342 & 1.0000 & 0.9831 & 0.8914 & 0.7131 \\
\hline 21 & 0.9001 & 0.7889 & 1.0000 & 0.9999 & 0.9648 & 0.5175 & 0.6191 & 0.6638 & 0.6700 & 0.7369 & 0.9542 & 0.8508 & 0.7180 & 0.8416 & 0.8601 & 1.0000 & 0.6868 & 0.7667 & 0.9311 & 0.7310 & 0.9453 & 0.8720 \\
\hline 22 & 0.9327 & 0.8080 & 0.9775 & 0.8680 & 0.7746 & 0.5100 & 0.5843 & 0.8170 & 0.7301 & 0.8503 & 0.9641 & 0.8428 & 0.7108 & 0.9186 & 0.9308 & 1.0000 & & 0.8241 & 0.9674 & 0.7752 & 0.9414 & 1.0000 \\
\hline Ave. & 0.8352 & 0.7230 & 0.9599 & 0.8805 & 0.7881 & 0.4398 & 0.5420 & 0.6859 & 0.6586 & 0.7424 & 0.8928 & 0.8030 & 0.6769 & 0.8248 & 0.8569 & 0.9771 & 0.6531 & 0.7425 & 0.8896 & 0.6799 & 0.8742 & 0.8409 \\
\hline
\end{tabular}


Table 4. The cross-efficiency of 22 Taiwanese commercial banks via Liang et al. [5].

\begin{tabular}{|c|c|c|c|c|c|c|c|c|c|c|c|c|c|c|c|c|c|c|c|c|c|c|}
\hline Bank & 1 & 2 & 3 & 4 & 5 & 6 & 7 & 8 & 9 & 10 & 11 & 12 & 13 & 14 & 15 & 16 & 17 & 18 & 19 & 20 & 21 & 22 \\
\hline 1 & 0.9327 & 0.8080 & 0.9776 & 0.8681 & 0.7747 & 0.5105 & 0.5843 & 0.8173 & 0.7306 & 0.8504 & 0.9641 & 0.8428 & 0.7109 & 0.9186 & 0.9309 & 1.0000 & 0.7709 & 0.8242 & 0.9680 & 0.7764 & 0.9414 & 1.0000 \\
\hline 2 & 0.8062 & 0.8496 & 1.0000 & 0.7653 & 0.7061 & 0.3149 & 0.5044 & 0.6320 & 0.5119 & 0.7662 & 0.8651 & 0.7627 & 0.7211 & 0.9036 & 0.9075 & 0.8610 & 0.5348 & 0.8481 & 0.6687 & 0.4204 & 0.8170 & 0.9663 \\
\hline 3 & 0.9296 & 0.8496 & 1.0000 & 1.0000 & 1.0000 & 0.5175 & 0.6192 & 0.8210 & 0.7066 & 0.9020 & 1.0000 & 0.8654 & 0.7767 & 1.0000 & 1.0000 & 1.0000 & 0.7388 & 0.8481 & 0.9344 & 0.7311 & 0.9453 & 1.0000 \\
\hline 4 & 0.9000 & 0.7889 & 1.0000 & 1.0000 & 1.0000 & 0.6306 & 0.6205 & 0.6781 & 0.7262 & 0.7368 & 0.9839 & 0.8541 & 0.7520 & 0.8731 & 0.8617 & 1.0000 & 0.7333 & 0.7666 & 1.0000 & 0.9831 & 0.9453 & 0.8719 \\
\hline 5 & 0.8356 & 0.7796 & 1.0000 & 1.0000 & 1.0000 & 0.6103 & 0.5969 & 0.5992 & 0.7076 & 0.7108 & 0.9058 & 0.8637 & 0.7132 & 0.7929 & 0.9405 & 1.0000 & 0.6384 & 0.7368 & 0.9617 & 0.9287 & 0.8936 & 0.7977 \\
\hline 6 & 0.8322 & 0.6649 & 0.8758 & 1.0000 & 0.9793 & 0.6306 & 0.6024 & 0.5872 & 0.6920 & 0.6218 & 0.8764 & 0.7863 & 0.6269 & 0.7041 & 0.7266 & 0.9454 & 0.7022 & 0.6338 & 1.0000 & 0.9831 & 0.8916 & 0.7122 \\
\hline 7 & 0.8973 & 0.7477 & 0.9563 & 1.0000 & 0.9592 & 0.5835 & 0.6205 & 0.6781 & 0.7139 & 0.7215 & 0.9425 & 0.8392 & 0.6875 & 0.8077 & 0.8277 & 1.0000 & 0.7333 & 0.7283 & 1.0000 & 0.8732 & 0.9433 & 0.8382 \\
\hline 8 & 0.7303 & 0.5957 & 0.9141 & 0.7474 & 0.6655 & 0.3391 & 0.4453 & 0.9050 & 0.7980 & 0.9419 & 0.7408 & 0.8295 & 0.5749 & 0.6963 & 1.0000 & 1.0000 & 0.6679 & 0.7193 & 0.9461 & 0.7053 & 0.7483 & 0.7738 \\
\hline 9 & 0.7992 & 0.6475 & 0.9124 & 0.7882 & 0.6986 & 0.4152 & 0.4919 & 0.8877 & 0.8058 & 0.8951 & 0.8112 & 0.8273 & 0.6074 & 0.7560 & 0.9494 & 1.0000 & 0.7358 & 0.7371 & 1.0000 & 0.8168 & 0.8141 & 0.8362 \\
\hline 10 & 0.7303 & 0.5957 & 0.9141 & 0.7473 & 0.6655 & 0.3391 & 0.4452 & 0.9050 & 0.7980 & 0.9419 & 0.7408 & 0.8295 & 0.5749 & 0.6962 & 1.0000 & 1.0000 & 0.6678 & 0.7193 & 0.9461 & 0.7053 & 0.7482 & 0.7738 \\
\hline 11 & 0.8445 & 0.7384 & 1.0000 & 0.9372 & 0.6270 & 0.2850 & 0.5029 & 0.5211 & 0.4888 & 0.5500 & 1.0000 & 0.7123 & 0.7767 & 1.0000 & 0.7129 & 1.0000 & 0.5282 & 0.7460 & 0.7443 & 0.3570 & 0.9209 & 0.8462 \\
\hline 12 & 0.5076 & 0.4353 & 1.0000 & 0.8981 & 0.9609 & 0.2191 & 0.3892 & 0.4685 & 0.5813 & 0.7384 & 0.5527 & 0.8654 & 0.5012 & 0.4191 & 1.0000 & 1.0000 & 0.3567 & 0.5418 & 0.7023 & 0.4043 & 0.5902 & 0.3967 \\
\hline 13 & 0.7861 & 0.6859 & 1.0000 & 0.8883 & 0.5305 & 0.2056 & 0.4673 & 0.3754 & 0.3841 & 0.3837 & 1.0000 & 0.6378 & 0.7767 & 1.0000 & 0.5796 & 0.9873 & 0.4215 & 0.6850 & 0.6447 & 0.2257 & 0.8989 & 0.7391 \\
\hline 14 & 0.8116 & 0.7115 & 1.0000 & 0.8379 & 0.5043 & 0.2351 & 0.4755 & 0.4418 & 0.4273 & 0.4605 & 1.0000 & 0.6687 & 0.7767 & 1.0000 & 0.6419 & 1.0000 & 0.4664 & 0.7164 & 0.6836 & 0.2757 & 0.9055 & 0.7934 \\
\hline 15 & 0.8329 & 0.7377 & 1.0000 & 0.8978 & 0.9606 & 0.3773 & 0.5145 & 0.9050 & 0.7980 & 0.9419 & 0.8622 & 0.8654 & 0.6751 & 0.8366 & 1.0000 & 1.0000 & 0.6716 & 0.8166 & 0.9461 & 0.7053 & 0.8480 & 0.9163 \\
\hline 16 & 0.9327 & 0.8248 & 1.0000 & 1.0000 & 1.0000 & 0.5835 & 0.6205 & 0.9050 & 0.8058 & 0.9419 & 1.0000 & 0.8654 & 0.7743 & 1.0000 & 1.0000 & 1.0000 & 0.7958 & 0.8397 & 1.0000 & 0.8732 & 0.9453 & 1.0000 \\
\hline 17 & 0.9322 & 0.7890 & 0.9574 & 0.8654 & 0.7689 & 0.5383 & 0.5841 & 0.8295 & 0.7530 & 0.8454 & 0.9590 & 0.8374 & 0.6968 & 0.9036 & 0.9166 & 1.0000 & 0.7958 & 0.8066 & 1.0000 & 0.8414 & 0.9404 & 0.9857 \\
\hline 18 & 0.8064 & 0.8496 & 1.0000 & 0.7654 & 0.7062 & 0.3151 & 0.5045 & 0.6322 & 0.5121 & 0.7663 & 0.8653 & 0.7628 & 0.7211 & 0.9036 & 0.9075 & 0.8611 & 0.5350 & 0.8481 & 0.6690 & 0.4207 & 0.8172 & 0.9664 \\
\hline 19 & 0.9322 & 0.7890 & 0.9574 & 1.0000 & 0.9835 & 0.6306 & 0.6205 & 0.8876 & 0.8058 & 0.8950 & 0.9590 & 0.8392 & 0.6968 & 0.9036 & 0.9494 & 1.0000 & 0.7958 & 0.8066 & 1.0000 & 0.9831 & 0.9433 & 0.9857 \\
\hline 20 & 0.8323 & 0.6650 & 0.8758 & 1.0000 & 0.9793 & 0.6306 & 0.6024 & 0.5883 & 0.6923 & 0.6226 & 0.8764 & 0.7863 & 0.6269 & 0.7046 & 0.7270 & 0.9454 & 0.7027 & 0.6342 & 1.0000 & 0.9831 & 0.8916 & 0.7131 \\
\hline 21 & 0.9001 & 0.7889 & 1.0000 & 1.0000 & 0.9649 & 0.5176 & 0.6192 & 0.6638 & 0.6700 & 0.7369 & 0.9542 & 0.8508 & 0.7180 & 0.8416 & 0.8601 & 1.0000 & 0.6868 & 0.7667 & 0.9311 & 0.7312 & 0.9453 & 0.8720 \\
\hline 22 & 0.9327 & 0.8354 & 1.0000 & 0.8850 & 0.8008 & 0.5100 & 0.5883 & 0.8170 & 0.7301 & 0.8622 & 0.9726 & 0.8489 & 0.7295 & 0.9399 & 0.9539 & 1.0000 & 0.7704 & 0.8450 & 0.9674 & 0.7752 & 0.9428 & 1.0000 \\
\hline Ave. & 0.8384 & 0.7354 & 0.9700 & 0.9041 & 0.8289 & 0.4518 & 0.5463 & 0.7066 & 0.6745 & 0.7651 & 0.9014 & 0.8110 & 0.6916 & 0.8455 & 0.8815 & 0.9818 & 0.6568 & 0.7552 & 0.8961 & 0.7045 & 0.8763 & 0.8538 \\
\hline
\end{tabular}


Table 5. Three different cross-efficiency scores for 22 commercial banks in Taiwan.

\begin{tabular}{cccc}
\hline Bank & Model (5) & Model (8) & Model (9) \\
\hline 1 & $0.8382(9)$ & $0.8352(9)$ & $0.8384(10)$ \\
2 & $0.7294(15)$ & $0.7230(15)$ & $0.7354(15)$ \\
3 & $0.9651(2)$ & $0.9599(2)$ & $0.9700(2)$ \\
4 & $0.8757(5)$ & $0.8805(5)$ & $0.9041(3)$ \\
5 & $0.7811(12)$ & $0.7881(12)$ & $0.8289(11)$ \\
6 & $0.4341(22)$ & $0.4398(22)$ & $0.4518(22)$ \\
7 & $0.5411(21)$ & $0.5420(21)$ & $0.5463(21)$ \\
8 & $0.6929(16)$ & $0.6859(16)$ & $0.7066(16)$ \\
9 & $0.6583(19)$ & $0.6586(19)$ & $0.6745(19)$ \\
10 & $0.7503(14)$ & $0.7424(14)$ & $0.7651(13)$ \\
11 & $0.8959(3)$ & $0.8928(3)$ & $0.9014(4)$ \\
12 & $0.8050(11)$ & $0.8030(11)$ & $0.8110(12)$ \\
13 & $0.6809(17)$ & $0.6769(18)$ & $0.6916(18)$ \\
14 & $0.8332(10)$ & $0.8248(10)$ & $0.8455(9)$ \\
15 & $0.8649(7)$ & $0.8569(7)$ & $0.8815(6)$ \\
16 & $0.9785(1)$ & $0.9771(1)$ & $0.9818(1)$ \\
17 & $0.6538(20)$ & $0.6531(20)$ & $0.6568(20)$ \\
18 & $0.7504(13)$ & $0.7425(13)$ & $0.7552(14)$ \\
19 & $0.8861(4)$ & $0.8896(4)$ & $0.8961(5)$ \\
20 & $0.6711(18)$ & $0.6799(18)$ & $0.7045(17)$ \\
21 & $0.8755(6)$ & $0.8742(6)$ & $0.8763(7)$ \\
22 & $0.8516(8)$ & $0.8409(8)$ & $0.8538(8)$ \\
\hline
\end{tabular}

The normalized cross-efficiency scores are presented in columns 2-4 of Table 6, and Shannon's entropies for the three models (i.e., $q=3$ ) are measured as $H_{1}=0.9951(\operatorname{Model}(5)), H_{2}=0.9952(\operatorname{Model}(8))$, and $H_{3}=0.9954$ (Model(9)), respectively. According to Step 4 of the algorithm, the associated degrees of diversification for the three models are $D_{1}=0.0049, D_{2}=0.0048$, and $D_{3}=0.0046$, respectively. With these values, we can easily calculate the degrees of importance as $w_{1}=0.3419, w_{2}=0.3348$, and $w_{3}=0.3233$, respectively. The composite cross-efficiency scores are then obtained by using Step 6 of the algorithm, with the results shown in the second-to-last column of Table 6.

Table 6. Normalized and composite cross-efficiency scores for 22 commercial banks in Taiwan.

\begin{tabular}{cccccc}
\hline Bank & Model (5) & Model (8) & Model (9) & $\boldsymbol{E}_{j}^{C^{*}}$ & Rank \\
\hline 1 & 0.0493 & 0.0492 & 0.0485 & 0.8373 & 9 \\
2 & 0.0429 & 0.0426 & 0.0426 & 0.7292 & 15 \\
3 & 0.0567 & 0.0566 & 0.0561 & 0.9650 & 2 \\
4 & 0.0515 & 0.0519 & 0.0523 & 0.8865 & 5 \\
5 & 0.0459 & 0.0464 & 0.0480 & 0.7989 & 12 \\
6 & 0.0255 & 0.0259 & 0.0261 & 0.4417 & 22 \\
7 & 0.0318 & 0.0319 & 0.0316 & 0.5431 & 21 \\
8 & 0.0407 & 0.0404 & 0.0409 & 0.6950 & 16 \\
9 & 0.0387 & 0.0388 & 0.0390 & 0.6636 & 19 \\
10 & 0.0441 & 0.0438 & 0.0443 & 0.7525 & 13 \\
11 & 0.0527 & 0.0526 & 0.0522 & 0.8966 & 3 \\
12 & 0.0473 & 0.0473 & 0.0469 & 0.8063 & 11 \\
13 & 0.0400 & 0.0399 & 0.0400 & 0.6830 & 18 \\
14 & 0.0490 & 0.0486 & 0.0489 & 0.8344 & 10 \\
15 & 0.0508 & 0.0505 & 0.0510 & 0.8676 & 7 \\
16 & 0.0575 & 0.0576 & 0.0568 & 0.9791 & 1 \\
17 & 0.0384 & 0.0385 & 0.0380 & 0.6546 & 20 \\
18 & 0.0441 & 0.0438 & 0.0437 & 0.7493 & 14 \\
19 & 0.0521 & 0.0524 & 0.0519 & 0.8905 & 4 \\
20 & 0.0394 & 0.0401 & 0.0408 & 0.6849 & 17 \\
21 & 0.0515 & 0.0515 & 0.0507 & 0.8753 & 6 \\
22 & 0.0501 & 0.0496 & 0.0494 & 0.8487 & 8 \\
\hline
\end{tabular}

Of the 22 commercial banks, based on their calculated composite cross-efficiencies, bank no. 16 is in first place, followed by banks nos. 3,11, 19, and 4 subsequently. This shows that combining the 
efficiency results of different cross-efficiency models gives more practical ranking results for decision makers compared with using each of cross-efficiency models respectively. For example, if a DMU has a strict maximum output value for any output item, then it may be measured as an efficient unit by some cross-efficiency evaluation models. Under this situation the model might decide the efficiency based upon one factor, and it seems to be not reasonable. This can be avoided by using the procedure for the combination of different cross-efficiencies proposed in this study.

\section{Conclusions}

Cross-efficiency evaluation is an effective approach for ranking DMUs. Nevertheless, different approaches measure cross-efficiency scores from different perspectives, and the efficiency rankings derived from evaluation models proposed in the literature may not be the same. In this case, we are hardly able to determine which method should be used. Besides, each model used for generating cross-efficiencies might have valuable viewpoints that we need to care about. To this end, this study applies the idea of Soleimani-Damaneh and Zarepisheh [14] to calculate Shannon's entropy of the obtained cross-efficiencies from different evaluation models. With the calculated composite cross-efficiency scores provided in this study, DMUs are fully ranked accordingly. A measure for estimating the importance degree of cross-efficiency evaluation models is provided as well. The example of commercial banks in Taiwan is used to illustrate the approach proposed in this paper, and the results derived indicate that the proposed approach is able to provide composite cross-efficiency scores for ranking DMUs effectively.

Funding: This research received no external funding.

Acknowledgments: The author thanks the reviewers for their constructive comments on an earlier version of this paper.

Conflicts of Interest: The author declares no conflict of interest.

\section{References}

1. Anderson, T.R.; Hollingsworth, K.B.; Inman, L.B. The fixed weighting nature of a cross-evaluation model. J. Prod. Anal. 2002, 17, 249-255. [CrossRef]

2. Sexton, T.R.; Silkman, R.H.; Hogan, A.J. Data envelopment analysis: Critique and extensions. In Measuring Efficiency: An Assessment of Data Envelopment Analysis; Silkman, R.H., Ed.; Jossey-Bass: San Francisco, CA, USA, 1986.

3. Doyle, J.R.; Green, R.H. Efficiency and cross efficiency in DEA: Derivations, meanings and uses. J. Oper. Res. Soc. 1994, 45, 567-578. [CrossRef]

4. Liang, L.; Wu, J.; Cook, W.D.; Zhu, J. Alternative secondary goals in DEA cross efficiency evaluation. Int. J. Prod. Econ. 2008, 113, 1025-1030. [CrossRef]

5. Liang, L.; Wu, J.; Cook, W.D.; Zhu, J. The DEA game cross efficiency model and its Nash equilibrium. Oper. Res. 2008, 56, 1278-1288. [CrossRef]

6. Ramón, N.; Ruiz, J.L.; Sirvent, I. Reducing differences between profiles of weights: a "peer restricted" cross-efficiency evaluation. OMEGA 2011, 39, 634-641. [CrossRef]

7. Wang, Y.M.; Chin, K.S.; Jiang, P. Weight determination in the cross-efficiency evaluation. Comput. Ind. Eng. 2011, 61, 497-502. [CrossRef]

8. Wu, J.; Sun, J.; Liang, L. Cross efficiency evaluation method based on weight-balanced data envelopment analysis model. Comput. Ind. Eng. 2012, 63, 513-519. [CrossRef]

9. Wu, J.; Chu, J.F.; Sun, J.S.; Zhu, Q.Y.; Liang, L. Extended secondary goal models for weights selection in DEA cross-efficiency evaluation. Comput. Ind. Eng. 2016, 93, 143-151. [CrossRef]

10. Liu, S.T. A DEA ranking method based on cross-efficiency intervals and signal-to-noise ratio. Ann. Oper. Res. 2018, 216, 207-232. [CrossRef]

11. Oukil, A. Ranking via composite weighting schemes under a DEA cross-evaluation framework. Comput. Ind. Eng. 2018, 117, 217-224. [CrossRef] 
12. Al-Siyabi, M.; Amin, G.R.; Bose, S.; Al-Masrooriet, H. Peer-judgment risk minimization using DEA cross-evaluation with an application in fishery. Ann. Oper. 2019, 274, 39-55. [CrossRef]

13. Liu, W.; Wang, Y.M.; Lv, S. An aggressive game cross-efficiency evaluation in data envelopment analysis. Ann. Oper. 2017, 259, 241-258. [CrossRef]

14. Soleimani-Damaneh, M.; Zarepisheh, M. Shannon's entropy for combining the efficiency results of different DEA models: Method and application. Expert Syst. Appl. 2009, 36, 5146-5150. [CrossRef]

15. Xie, Q.; Dai, Q.; Li, Y.; Jiang, A. Increasing the discriminatory power of DEA using Shannon's entropy. Entropy 2014, 16, 1571-1585. [CrossRef]

16. Qi, X.G.; Guo, B. Determining common weights in data envelopment analysis with Shannon's entropy. Entropy 2014, 16, 6394-6414. [CrossRef]

17. Wang, L.; Li, L.; Hong, N. Entropy cross-efficiency model for decision making units with interval data. Entropy 2016, 18, 358. [CrossRef]

18. Lu, T.; Liu, S.T. Ranking DMUs by comparing DEA cross-efficiency intervals using entropy Measures. Entropy 2016, 18, 452. [CrossRef]

19. Zeleny, M. Multiple Criteria Decision Making; McGraw-Hill: New York, NY, USA, 1982.

20. Charnes, A.; Cooper, W.W.; Rhodes, E. Measuring the efficiency of decision making units. Eur. J. Oper. Res. 1978, 2, 429-444. [CrossRef]

21. Charnes, A; Cooper, W.W. Programming with linear fractional functional. Nav. Res. Logist. Q. 1962, 9, 181-186. [CrossRef]

22. Shannon, C.E. A mathematical theory of communication. Bell Syst. Tech. J. 1948, 27, 379-423. [CrossRef]

(C) 2019 by the author. Licensee MDPI, Basel, Switzerland. This article is an open access article distributed under the terms and conditions of the Creative Commons Attribution (CC BY) license (http://creativecommons.org/licenses/by/4.0/). 